\title{
Ein neues Therapie-Konzept validiert die besonderen Bedürfnisse geflüchteter Kinder und Jugendlicher: START
}

\author{
START - Stress-Traumasymptoms-Arousal-Regulation-Treatment
}

\author{
Andrea Dixius · Eva Möhler
}

Eingegangen: 11. März 2017 / Angenommen: 28. April 2017 / Online publiziert: 23. Mai 2017

C Der/die Autor(en) 2017. Dieser Artikel ist eine Open-Access-Publikation.

Zusammenfassung Die besondere Situation psychisch belasteter und geflüchteter Kinder und Jugendlicher erfordert neue Konzepte in der psychotherapeutischen Versorgung.

Geflüchtete Kinder leiden häufig an psychischen und körperlichen Symptomen und sind durch traumatische Erlebnisse nachhaltig belastet. Das strukturierte und manualisierte Kurztherapiemanual „START“ Stress-Traumasymptoms-Arousal-Regulation-Treatment wurde im Rahmen der praktischen Arbeit mit unbegleiteten minderjährigen Flüchtlingen in Clearing- und Erstaufnahmekontexten und der klinischen Versorgung der Kinder- und Jugendpsychiatrie entwickelt. Interventionen aus der Dialektisch-Behavioralen Therapie, Psychotraumatherapie und Alptraumtherapie werden in START integriert.

Dabei zielen die Interventionen primär auf eine rasche Stabilisierung und Arousal-Regulation bei emotionaler Belastung und ausgeprägtem Stresserleben. Im zweiten Schritt fokussiert START auf Förderung von Selbstwirksamkeit und die Stärkung von Schutzfaktoren. Der folgende Beitrag gibt einen kurzen Überblick zur Situation der geflüchteten Kinder und Jugendlichen und beschreibt das START-Konzept hinsichtlich klinischer Implikationen und auch der Limitationen.

Schlüsselwörter Erststabilisierung - Arousal-Regulation · Interkulturelle Behandlung · Stressregulation

Diese Arbeit ist Teil des Leitthemas „Psychotherapie mit Kindern und Jugendlichen“.

A. Dixius $(\bowtie) \cdot$ E. Möhler $(\bowtie)$

SHG Kliniken für Kinder- und Jugendpsychiatrie,

Psychotherapie und Psychosomatik,

Dr.-Ottmar-Kohler-Str. 2, 55743 Idar-Oberstein, Deutschland

a.dixius@io.shg-kliniken.de; e.moehler@sb.shg-kliniken.de
A new therapyconcept validating the special needs of refugeed children and adolescents START - Stress-Traumasymptoms-ArousalRegulation-Treatment

Summary Recent research shows that unaccompanied refugeed minors frequently suffer from sleeping disorder, night mares, hyperarousal and emotion dysregulation due to severe traumatic experiences. Furthermore many are in acute stress during the clearing process on arrival in Germany.

START was developed to reduce stress and improve psychosocial functioning in this population. It is a short manualized structured intervention for crisis intervention and stabiliziation of children and adolescents suffering from intense posttraumatic tension or desperation.

Compounds of START are derived from elements of dialectic behavioral therapy and traumafocused cognitive behavioral therapy for children.

The manual can be used by psychotherapists, social workers, physicians, school psychologists as well as qualified caretakers, given the highly self-instructive character of the instrument. In clinical settings, children of all cultural backgrounds can and should be included in the group treatment.

Its efficacy and effectiveness is currently target of a standardized pre-post evaluation. Clinical implications as well as limitations of the concept are discussed.

Keywords Arousal-regulation · Emotional stabilization · Intercultural treatment $\cdot$ Stressregulation 
Hintergrundinformationen zur Situation von geflüchteten minderjährigen Kindern und Jugendlichen

Weltweit befinden sich derzeit mehr als 60 Mio. Menschen auf der Flucht. Die meisten Flüchtlinge kommen aus Syrien, Afghanistan und Somalia. In der ersten Jahreshälfte 2015 wurden in der Bundesrepublik Deutschland mit 159.900 weltweit die meisten Neuanträge auf Asyl gestellt. Etwa die Hälfte der Flüchtlinge weltweit sind Kinder und Jugendliche unter 18 Jahren. Ende Januar 2016 ist die Zahl der unbegleiteten minderjährigen Flüchtlinge (UMF) in Deutschland auf über 60.000 gestiegen; Hauptherkunftsländer der UMF waren 2015 Afghanistan, Syrien, Irak, Eritrea und Somalia (BAMF 2016). Die Anamnesen der Minderjährigen sind gekennzeichnet von körperlicher und sexueller Gewalt, Krieg und Trennung bzw. Verlust von Familienmitgliedern und insbesondere die unbegleiteten Flüchtlinge weisen eine relativ hohe subjektive Belastung durch Symptome einer PTBS auf (Hodes et al. 2008).

Witt et al. (2015) konnten in einer Übersichtsarbeit zeigen, dass die Prävalenzzahlen für psychische Auffälligkeiten bei UMF zwischen 20 und 81,5\% liegen. Die erheblichen Schwankungen sind in erster Linie auf die eingesetzten Methoden der unterschiedlichen Studien zurückzuführen. Die Autoren kommen zu dem Schluss, dass UMF in Bezug auf psychische Auffälligkeiten eine Hochrisikogruppe darstellen und eine hohe Prädisposition zur Entwicklung von Traumafolgestörungen haben.

Die Vielzahl der traumatischen Erfahrungen können zudem als Prädiktor für das Risiko eine posttraumatische Belastungsstörung (PTBS) zu entwickeln (Mollica et al. 1998) herangezogen werden. Minderjährige Flüchtlinge sind sicherlich als hoch vulnerable Gruppe zu sehen, mit der Gefährdung eine psychiatrische Erkrankung zu entwickeln (Huemer et al. 2009), was durch das Fehlen von Schutzfaktoren zusätzlich begünstigt werden kann.

\section{Lula, 16 Jahre}

„Ich habe mich seit ein paar Tagen nicht gut gefühlt. Ich hatte mich mehrmals erbrochen und hatte Bauchschmerzen. Ich habe mich schwach gefühlt. Meine Betreuerin aus der Wohngruppe hatte mich dann zum Arzt und in die Kinderklinik gefahren. Ich hatte sehr große Angst. Sie sagten mir in der Klinik, dass ich im fünften Monat schwanger sei. Ich hatte dies nicht gewusst und fühlte mich zuerst taub, dann nur noch verzweifelt. Schlimme und furchtbare Erinnerungen kamen plötzlich in meinen Kopf, was die ganzen Männer mir angetan haben. Ich wollte nur noch sterben ...“

Flucht, Folter, Zwangsarbeit, Vertreibung, Vergewaltigung, Gefahr durch Kriegswirren, lebensbedrohliche Situationen und massive Entbehrungen vielfältiger Art über einen meist langen Zeitraum führen häufig $\mathrm{zu}$ schweren psychischen Beeinträchtigungen und massiven Einschränkungen des allgemeinen Funktionsniveaus (Moehler et al. 2015; Dixius und Moehler 2017).

Die Organisation „Save The Children“ (2017) schildert in ihrer Studie zur Situation von syrischen Kindern, dass die permanente Bedrohung durch Kriegserlebnisse bei vielen Kindern $\mathrm{zu}$ einem sogenannten „toxischen Stress“ führen kann und sich zunehmend Symptome von Bettnässen, Selbstverletzungen, Suizidversuchen und aggressivem oder sich selbst isolierendem Verhalten beobachten lassen.

Bleiben diese Beeinträchtigungen unbehandelt, dann kann davon ausgegangen werden, dass die psychische und körperliche Gesundheit von Kindern und Jugendlichen auch dauerhaft belastet bleibt (Miller et al. 2011).

\section{Exkurs: Trauma und Traumafolgen, PTBS}

Die Posttraumatische Belastungsstörung (PTBS) ist eine verzögerte oder protrahierte Reaktion auf ein belastendes Ereignis oder Situation kürzerer oder längerer Dauer, mit außergewöhnlicher Bedrohung oder katastrophenartigem Ausmaß, die bei fast jedem eine tiefe Verzweiflung hervorrufen würde.

Das emotionale Erleben von Personen mit PTBS ist häufig begleitet durch intensive und bisweilen überflutende Emotionen wie Angst, Schuld, Scham, Trauer, Ärger sowie auch von emotionaler Taubheit geprägt. Beispielhaft einige weitere weitere Symptome: Entfremdungsgefühle, Dissoziationen, Flashbacks, sozialer Rückzug, Symptome autonomer Übererregung z. B. eine erhöhte Reaktionsbereitschaft, Hypervigilanz und Hyperarousal, starke Schreckreaktionen, Reizbarkeit, Konzentrationsprobleme und Schlafstörungen, Alpträume, emotionaler Dysregulation selbstverletzendem Verhalten, Suizidgedanken und -versuch.

Beeinträchtigte Bereiche und Konsequenzen auf das Verhalten und Erleben nach Traumatisierung

- Bindung

- Affektregulation

- Biologische Aspekte

- somatoforme Beschwerden

- Dissoziation (Intrusionen, Flashbacks)

- Verhaltenssteuerung/-kontrolle

- Suizidalität

- Selbstverletzungen

- Kognition

- Selbstkonzept

- körperliche Erkrankungen (z. B. coronare Erkrankungen, Infekte, Beeinträchtigung des Immunsystems mit schweren Folgeerkrankungen)

Die Ausprägung von Traumafolgen ist auch vom Entwicklungsstand und vom Alter des Kindes oder des Jugendlichen abhängig. 
Ahmad, 17 Jahre

„Auf meiner Flucht musste ich oft draußen schlafen, wir wurden auch überfallen. Seitdem fällt es mir noch schwerer zu schlafen. Ich bin manchmal total müde. Ich habe Angst zu schlafen, die Augen zu zumachen. Dann sehe ich immer wieder wie mein Bruder getötet wird. Ich höre meine verzweifelte Mutter. Manchmal ist dies auch plötzlich da, ich kann nicht schlafen und ich kann nicht an meinen Bruder denken. Immer wieder kommen die schrecklichen Erinnerungen. Ich halte das nicht aus...".

Krüsmann und Müller-Cyran (2005) kommen zu dem Schluss, dass frühe Interventionen für Menschen hilfreich sind, die potentiell traumatisierenden Ereignissen ausgesetzt waren. Dabei sollte sich die Intervention an folgenden Zielen orientieren: Die Intervention muss Übersicht über die Lage verschaffen; Stabilisierung unterstützen und sichere Räume schaffen; die eigene Situation besser strukturieren sowie innere Ressourcen fördern und Wiederherstellung der Handlungsfähigkeit fördern. Stabilisierende und selbstwirksamkeitsfördernde Interventionen erscheinen auf dieser Grundlage durchaus sinnvoll (Krüsmann 2003). Besonders unter dem Aspekt der Resilienzförderung und um einem pathologischen langfristigen Verlauf abzuschwächen und einer weiteren Chronifizierung entgegen zu wirken, erscheinen frühe Interventionen sinnvoll und als sekundäre Prävention wirkungsvoll (Bryant et al. 1998).

Praxiserfahrungen und Hintergrundinformation zur Erstversorgung geflüchteter Kinder und Jugendlicher im Saarland

Alle unbegleiteten minderjährigen geflüchteten Kinder und Jugendliche im Saarland, werden zunächst im Vor-Clearinghaus aufgenommen und registriert. Seit Februar 2016 wurden 562 Kinder und Jugendliche aufgenommen.

Das Vor-Clearinghaus in Tholey ist eine Erstaufnahmeeinrichtung für unbegleitete minderjährige Flüchtlinge und wurde im Februar 2016 gegründet. Damit wurde ein bundesweites Pilotprojekt für die Erstversorgung von unbegleiteten minderjährigen Ausländern (umA) geschaffen. Die Kinder- und Jugendlichen erhalten medizinische, pädagogische und therapeutische Unterstützung in dieser Clearingphase. Nach dem sogenannten „Königsteiner Schlüssel“ werden sie dann in der Regel in pädagogische Einrichtungen der Jugendhilfe nach Rheinland-Pfalz verteilt. Der Königsteiner Schlüssel gibt vor, wie viele Asylsuchende die einzelnen Bundesländer in Deutschland aufnehmen müssen. Die Quote errechnet sich zu zweidrittel aus Steuereinnahmen und zu einen Drittel aus der Bevölkerungszahl des jeweiligen Bundeslandes. Die Berechnung erfolgt jährlich neu und dient einer angemessen Verteilung der Asylsuchenden innerhalb Deutschlands (BAMF 2017).
Die meisten Jugendlichen schilderten bereits im Clearingkontext enorme psychische Beeinträchtigungen mit traumaassoziierten Inhalten. Viele Kinder und Jugendliche waren demnach einer sequentiellen Traumatisierung ausgesetzt. Peritraumatische Stressoren wie Lebensgefahr sowie Ressourcenverluste, mangelnde soziale Unterstützung und weitere belastende Lebensereignisse und prätraumatische psychische Morbidität begünstigten die Entwicklung einer PTBS. „Misstrauen“ und der Verlust von Kontrolle auf der Grundlage von erlebten „man-made-desaster" führen oft zu dysfunktionalen Verhaltensweisen und zu akuten, psychischen Krisen. Hinzu kommen zwischenmenschliche Belastungen wie die intensive Beschäftigung hinsichtlich der Sicherheit von Familienmitgliedern, anderen geliebten Personen oder auch Heimweh und Trennungsschmerz. Die Unsicherheit, wie es weitergehen würde, war für alle Jugendlichen verständlicherweise ein Thema.

Myogelosen, erosive Gastritis, Spannungskopfschmerzen und Muskelschmerzen waren die häufigsten beschrieben somatischen Beschwerden. Psychische Belastungen zeigten sich in Ängsten, Traurigkeit und Niedergeschlagenheit, Dissoziationen, Schlafstörungen, Alpträumen reduzierte Stressregulation, selbstverletzendes Verhalten und suizidale Krisen bei den Betroffenen. Alkoholabusus und Substanzkonsum werden als „dysfunktionale“ Regulationsprozesse bei emotionalem Stress eingesetzt.

In der klinischen Versorgung der Kinder- und Jugendpsychiatrie Kleinblittersdorf (Saarland) und IdarOberstein (Rheinland-Pfalz) wurden seit 2015 minderjährige Flüchtlinge mit schweren psychopathologischen Belastungen aufgenommen.

Viele geflüchtete Jugendliche hatten Angst die Kontrolle über ihre aktuelle Lebenssituation zu verlieren und hatten Misstrauen gegenüber einer psychiatrischen Behandlung. Auf dem Hintergrund der erlebten und durch Menschen verursachten Traumata macht dies durchaus Sinn und ist nachvollziehbar und verstehbar. Psychiatrie wird oft als Stigma psychisch krank zu sein empfunden und widerspricht damit identitätsstiftenden Werten der Jugendlichen und löst bei vielen das Gefühl der Scham aus. Mit dem Ziel Jugendliche wieder ,in self-control“ zu bringen, scheint die Entwicklung von neuen kurzen Behandlungskonzepten zur Erststabilisierung durchaus notwendig. Zudem gilt es Drehtür-Psychiatrieaufenthalte $\mathrm{zu}$ minimieren und Selbstwirksamkeit in Krisensituationen und Schutzfaktoren bei den Betroffenen zu fördern.

\section{Die Entwicklung von START}

START wurde auf der Grundlage von Praxiserfahrungen in Clearingkontexten und der klinischen Versorgung in der Kinder- und Jugendpsychiatrie entwickelt.

Wichtig erschien der Einsatz eines strukturierten, niedrigschwelligen Kurzzeittherapiekonzepts, um psychischen Dekompensationen und akuten Be- 
Abb. 1 Stressregulation und die Funktionen von Skills im Rahmen von START

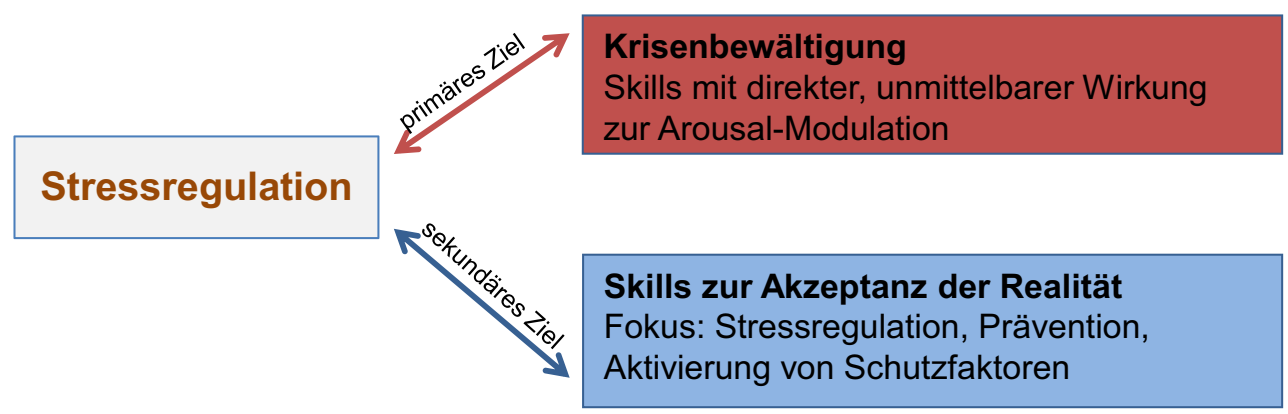

lastungsreaktionen sowie andauerndem Stresserleben vorzubeugen. Zudem zeigte die klinische Erfahrung, dass Kinder und Jugendliche in akuten Krisen aufgrund der Schwere ihrer Belastungen oftmals nicht in der Lage sind, sich gleich für eine aufarbeitende Behandlung oder langfristige Psychotherapie zu entscheiden, die auch nicht in allen Fällen notwendig erscheint. In der Praxisarbeit erwiesen sich Skills zur Stressregulation und Interventionen zur Selbstberuhigung wie PMR als hilfreich und stützend.

Die Manualisierung von START folgte nach dem trial and error-Prinzip: Skills und Emotionsregulationstechniken, die von den UMF gut angenommen werden konnten und als hilfreich erlebt wurden, wurden als Arbeitsblätter formuliert und in arabisch dari und englisch übersetzt. Während der Entwicklung des Manuals wurde die Wirksamkeit der Interventionen und Skills anhand der klinischen Beobachtung und der Rückmeldungen der Jugendlichen stetig reflektiert.

Mit den Interventionen und Skills soll in erster Linie eine rasche Stabilisierung und Arousal-Regulation erreicht werden, die es den Jugendlichen ermöglicht Krisen und leidvolle Situationen durchzustehen (Abb. 1). Im zweiten Schritt wird auf die Förderung von Selbstwirksamkeit, auf Prävention und auf die Stärkung von Schutzfaktoren fokussiert.

Stressregulationsskills, können emotionalen Stress und physiologisches Hyperarousal regulieren. Häufig können besonders Skills wie starke Sinnesreize, Kältereize oder Sport und Bewegung eine unmittelbar wahrnehmbare Reduktion des Hochstresserlebens beeinflussen. Dies unterstützt den Prozess die Eigenkontrolle des Verhaltens wieder zu erlangen und sich aktiv emotional und kognitiv beruhigen zu können, um so Krisen besser zu überstehen.

Des Weiteren ist es wichtig Selbstwirksamkeit $\mathrm{zu}$ fördern, dies vor allem unter präventiven und reslienzfördernden Gesichtspunkten, aber auch unter dem Aspekt, dass die durch Migration und Flucht erlebte Abhängigkeit zu einer narzisstischen Kränkung führen könnte, die das Selbstkonzept belastet - die Selbstwirksamkeit hinsichtlich der Regulation emotionaler Zustände hingegen, könnte das angeschlagene Selbstkonzept stabilisieren.

Resilienz bezieht sich damit nicht nur auf die Abwesenheit von psychischen Störungen, sondern auch auf den Erwerb und Erhalt altersangemessener Fähigkei- ten und Kompetenzen der normalen kindlichen Entwicklung trotz risikoreicher Lebensumstände (Rutter 2000). Im Vergleich zu früheren Ansätzen wird heute davon ausgegangen, dass Resilienz kein angeborenes Persönlichkeitsmerkma, sondern vielmehr eine Kapazität ist. die im Verlauf der Entwicklung im Kontext der Kind-Umwelt-Interaktion erworben wird (Rutter 2000). Das Resilienzparadigma beinhaltet in besonderem Maße die Sichtweise des Kindes als aktivem „Bewältiger" und „Mitgestalter seines eigenen Lebens“ zum Beispiel durch den effektiven Gebrauch seiner internen und externen Ressourcen. Dabei wird aber auch betont, dass Kinder sich natürlich nicht selbst dauerhaft „resilient machen können“, sondern hierzu maßgeblicher Hilfe und Unterstützung bedürfen.

Empirische Forschungen (Rutter und Sroufe 2000) haben herausgefunden, dass sich resiliente Kinder und Jugendliche durch folgende Merkmale auszeichnen:

- Problemlösefähigkeiten

- eine hohe Sozialkompetenz (Kontaktfähigkeit, soziale Perspektivenübernahme und Empathie)

- die Fähigkeit zur Selbstregulation

- ein aktives und flexibles Bewältigungsverhalten (z. B. die Fähigkeit, sich aktiv Hilfe zu holen oder sich von einer dysfunktionalen Familiensituation innerlich zu distanzieren)

- eine optimistische, zuversichtliche Lebenseinstellung

- eine internale Kontrollüberzeugung und ein realistischer Attribuierungsstil

- ein hohes Selbstwertgefühl sowie Selbstvertrauen in die eigenen Fähigkeiten und Selbstwirksamkeitsüberzeugungen

Auch hier setzt START an. Insbesondere bei der Förderung der Fähigkeit zur biologischen und emotionalen Selbstregulation durch Reduktion des traumabedingten Hyperarousal. Skills und Affektregulationsstrategien werden durch die internalen Kontrollüberzeugungen und Selbstwirksamkeitsüberzeugungen verstärkt, was insbesondere bei traumatisierten Menschen wichtig ist.

Die Gewalterfahrungen der Betroffenen kann das Selbstkonzept dahingehend verändern, dass sie sich als ohnmächtig und ausgeliefert empfinden - zum Beispiel dann auch den eigenen Gefühlen gegenüber. 
Abb. 2 Einleitung zu,Deine Persönliche Skillsliste“

\section{Info-/Übungsblatt}

\section{Deine Persönliche Skillsliste \\ Einleitung/ Beschreibung \\ (START-Modul 3 - S.42)}

Heute möchten wir mit dir zusammen deine persönliche Skillsliste zusammenstellen.

Deine persönliche Skillsliste soll dir helfen, dich an Skills zu erinnern, wenn du unter Hochstress kommst oder auch Skills berücksichtigen, um positive Gefühle und Gedanken zu fördern.

Wichtig ist, dass du besonders in hoher Anspannung, auf deinem Spannungsbogen (siehe ÜB aus Modul 2) über 70, so lange deine Skills aus dem roten Hochstressbereich anwendest, bis du wieder Kontrolle über dein Handeln hast, deine Gefühle wieder ausgeglichener sind und deine Gedanken wieder klarer.

Um dies zu erleichtern, werden wir heute für den Hochstress-Bereich auch eine Skillskette von 3 bis 4 Skills mit dir zusammenstellen.

\section{Einfach ausprobieren!!!}

Es ist wichtig die Fähigkeit zu stärken, sich nicht als Opfer der eigenen Stimmungsschwankungen zu erleben, sondern diesen selbstwirksam entgegenzutreten. Dadurch können sie nicht nur mit den vergangenen Belastungen besser umgehen, sondern auch zukünftigen Stressfaktoren resilienter begegnen.

START integriert und adaptiert Elemente zur Stressregulation, Achtsamkeit und zur Selbstfürsorge, zur Anspannungs- und Emotionswahrnehmung aus der Dialektisch Behavioralen Therapie (Linehan 2015; Rathus und Miller 2015; Bohus undWolf 2011). Entspannungs- und Stabilisierungstechniken aus der Traumafokussierten-Kognitiven Verhaltenstherapie (Cohen et al. 2009) sowie Hilfen im Umgang mit Alpträumen angelehnt an das Konzept der Alptraumtherapie nach Thünker und Pietrowsky (2011).

Skills werden von Linehan (1996b) als kognitive, emotionale und handlungsbezogene Reaktionen definiert, die kurz- und langfristig positive Erlebnisse fördern und negative reduzieren können. Jeder Mensch macht intuitiv regelmäßig Skills, in der DBT wird der bewusste Einsatz von Skills als Alternativfertigkeiten - anstelle von dysfunktionalen Verhaltensweisen wie z. B. Selbstverletzungen - geübt. Neben dem Skillstraining werden weitere Methoden der kognitiven Verhaltenstherapie eingesetzt. Module des Skillstrainings in der DBT-A (Rathus und Miller 2015) sind Achtsamkeit, Stresstoleranz, Emotionsregulation, zwischenmenschliche Fertigkeiten und Skills zum Thema Selbstwert und "goldener Mittelweg“.
START adaptiert Skills aus den Bereichen Achtsamkeit, Stressregulation und Basiselemente der Emotionsregulation. Dabei werden Skills unter Berücksichtigung der besonderen Lebenssituation der geflüchteten Jugendlichen geübt, hier ist die einfache Anwendung der Skills genauso wichtig wie deren Verfügbarkeit im Alltag der Jugendlichen. Eine individuelle Anpassung der Skills in Form einer „persönlichen Skillsliste“ (Abb. 2 und 3) wird zusammen mit den Jugendlichen erarbeitet, was durch den Einsatz von prägnanten Bildmaterialien erleichtert wird. Skills als adaptive Fertigkeiten zur Krisenbewältigung, zum Umgang mit Stress und mit belastenden Gefühlen können die Jugendlichen in Folge auf ihre Wirksamkeit in den Sitzungen und im Alltag erproben. Zudem werden Übungen zur Wahrnehmung von positiven Erfahrungen und Emotionen gezielt berücksichtigt.

Die Skillsliste erinnert im Alltag daran, welche Skills in den verschiedenen Anspannungsbereichen Unterstützung bieten können.

Grundsätzlich basiert die Intervention auf dem Grundgedanken, dass durch das aktive Anwenden von Skills die Selbstwirksamkeit und das Erleben von Kontrolle und Steuerung des eigenen Verhaltens verbessert werden kann (Bohus und Wolf 2011) Um den kulturintegrativen Aspekt in START zu fördern, nehmen im Gruppensetting Kinder und Jugendliche aus unterschiedlichen kulturellen Kontexten gemeinsam teil. Integration als zwischenmenschliche Erfahrung ist umso wichtiger, da häufig maladaptive Verhaltens- 


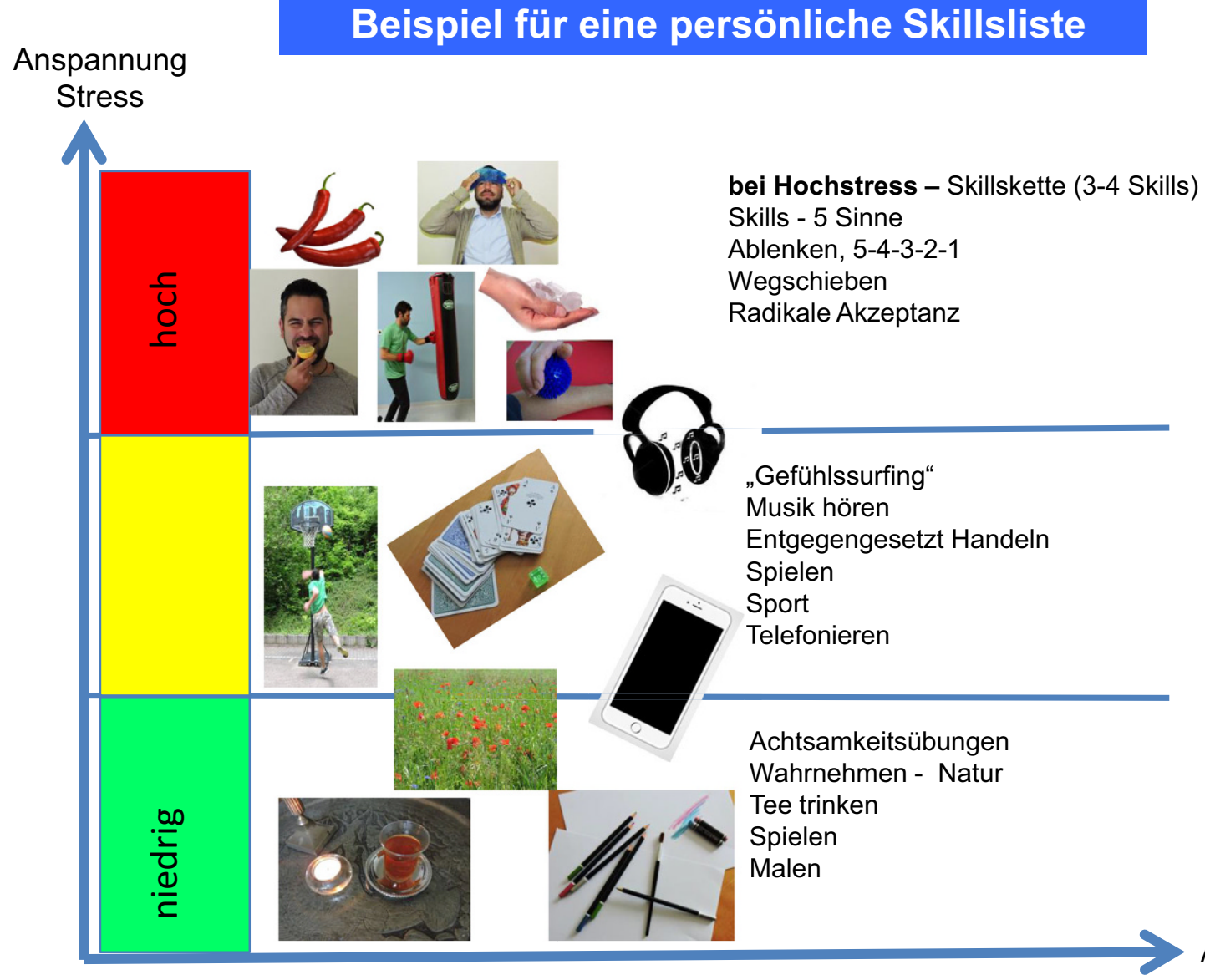

Anwendung

Abb. 3 Exemplarische Darstellung: „Deine Persönliche Skillsliste“

muster die Integration in psychosozialen Kontexten wie Schule, Wohngruppen und in Peer-Gruppen erschweren können. Mitunter führen gerade Momente in der Betreuungssituation, etwa Anforderungen an neue Regeln, die Anpassung an neue Werte und Normen bei den Kindern und Jugendlichen im pädagogischen und gesellschaftlichen Kontext zur Aufrechterhaltung von Gefühlen des „Ausgeliefertseins“.

Die expositionsbasierte oder narrative Aufarbeitung von traumatischen Erfahrungen ist expliziert kein Schwerpunkt in START, sondern primär die psychische und emotionale Stabilisierung durch Stressregulation. Insbesondere auch hinsichtlich der Selbstwirksamkeitsförderung kann START als ein Resilienzförderungsprogramm verstanden werden, das auch präventiv bei noch eher wenig symptomatischen Jugendlichen angewendet werden kann, um ein Abgleiten in Suchtentwicklungen oder andere Störungen, die aus der Verdrängung massivster Traumata resultieren vorzubeugen. So wird auch auf die Stärkung der Wahrnehmung von positiven Emotionen, die Orientierung auf Ziele und letztlich auch self-compassion (Gilbert 2013) in den Übungen ganz im dialektischen Sinne geachtet.

\section{Basisinformationen zu START}

Skills

Skills sind Fertigkeiten, die kurzfristig und langfristig helfen können. Skills können zum Beispiel Atementspannungstechniken und PMR sein, positive Gedanken, beruhigende Vorstellungen, sensorische Reize, Sport, Tanzen, Bewegung, Singen, Musik hören, Ablenkungsübungen bei Hyperarousal, Übungen zum Wahrnehmen von Gefühlen, antidissoziative Übungen, Übungen zur „Akzeptanz der Realität“.

Die START - Module konzentrieren sich vorwiegend auf Stressregulationskills und Achtsamkeitsskills sowie Skills zur Entspannung und Selbstberuhigung, compassion, Wahrnehmung von Emotionen und Förderung der Selbstwirksamkeit.

\section{Achtsamkeit}

Das Konzept der Achtsamkeit ist eine besondere Form der Aufmerksamkeit, die sich von alltäglichen, automatisch ablaufenden Wahrnehmungsprozessen unterscheidet, weil sie absichtsvoll, nicht wertend und auf das bewusste Erleben des aktuellen Augenblicks gerichtet ist. Achtsamkeit soll die Wahrnehmung verbessern, um aktivierte Gefühle und Gedanken besser 
wahrzunehmen und im weiteren Schritt auch frühzeitig regulieren zu können. Achtsamkeitsübungen sind bei zahlreichen psychischen und körperlichen Beschwerden hilfreich. Sich auf den eigenen Atem $\mathrm{zu}$ konzentrieren oder den Köper wahrzunehmen können Achtsamkeitsübungen sein, aber auch spielerische Übungen, die Konzentration und die Wahrnehmung des Augenblicks erfordern.

\section{Stressregulation}

Die Übungen zur Stressregulation zielen darauf ab, mit Hilfe von Skills oder Fertigkeiten selbstwirksam Krisen und Hochstressphasen oder leidvolle Situationen abzuschwächen und zu überstehen. Diese Fertigkeiten sind wichtig, da sich viele belastende Situationen nicht verändern lassen.

Ein Beispiel: Zur Unterbrechung von potentiellen, dissoziativen Zuständen, Intrusionen, flashbacks oder angespannten emotionalen Zuständen scheinen starke sensorische Reize und Bewegung und Sport geeignet zum Beispiel auf einer Chili kauen, Wasabi-Paste schmecken, Geruchsreize, Musik hören, Sport und Bewegung. Im Hochstressbereich erweisen sich zudem Kältereize wie kaltes Wasser an Nase und Mund oder ins Gesicht, mit kaltem Wasser duschen, sog. „diving skill“ als hilfreich. Auf diese Weise wird ein alter phylogenetischer Reflex - „Tauchreflex“ - ausgelöst, der den Nervus vagus aktiviert (Bohus und Wolf 2011). Eine Reduzierung der physiologischen Anspannung kann dadurch unterstützt werden.

\section{Anwendung von START}

Für Fachleute stehen grundlegende Kurzbeschreibungen zu den Themen Trauma, Resilienz, Skills, Achtsamkeit, Stressregulation, Selbstberuhigungstechniken, Alpträume zur Verfügung und ein detaillierter Sitzungsleitfaden führt durch die einzelnen Module.

Grundsätzlich ist es günstig mit zwei Therapeuten eine START-Gruppe zu leiten. So ist es möglich sich auf die Gruppe einzulassen, die Übungen anzuleiten, aber auch bei Bedarf einzelnen Teilnehmern in Anspannungssituationen direkte Unterstützung anzubieten. Die Therapeuten stellen die Übungen vor und führen sie auch aktiv und modellhaft mit den Jugendlichen zusammen durch.

\section{Der Aufbau der einzelnen Module und Sitzungen folgt einem strukturierten Ablaufschema}

- Begrüßung und eine kurze Achtsamkeitsübung zu Beginn der Sitzung

- Spannungsbogen (aktuelle Anspannung zu Beginn der Sitzung)

- Achtsamkeitsübung

- Information zum Thema der Sitzung

- thematische Einheit und Praxisübungen
- kleine „Übungsaufgabe“ bis zur nächsten Therapiesitzung (z. B. Spannungsbogen führen und mindestens Skills ausprobieren)

- Achtsamkeits- oder Entspannungsübung

- Spannungsbogen (aktuelle Anspannung am Ende der Sitzung)

Der strukturierte Sitzungsablauf ermöglicht den Jugendlichen sich rasch $\mathrm{zu}$ orientieren und sich aktiv in die Sitzungen einzubringen und diese mitzugestalten. Zum Beispiel können die Jugendlichen die Klangschale zur Achtsamkeitsübung betätigen, an der Flipchart die Anspannung und die aktuelle emotionale Verfassung in den Spannungsbogen von allen Teilnehmern erfragen und eintragen. Auch eigene neue Skills und Erfahrungsbeispiele von angewendeten Fertigkeiten können eingebracht werden. Die aktive Mitgestaltung der Sitzungen ist von zentralem Interesse und kann das Commitment und selbstwirksames Erleben und Handeln bei den Jugendlichen fördern.

Das START-Manual (Abb. 4) besteht aus fünf Modulen und kann im Gruppen- oder Einzelsetting durchgeführt werden. Das Manual ist in leicht verständlicher Sprache verfasst und bietet einen niedrigschwelligen und teilweise spielerischen Zugang. Die Informationen zu den Übungen liegen in arabisch, dari, deutsch und englisch vor und sind mit unterstützendem Bildmaterial ausgestattet. Für die vereinfachte praktische Durchführung stehen zudem neben übersetzten Printmaterialien und auch mehrsprachige Audiodateien auf der zum Manual gehörenden DVD zur Verfügung.

Das Gruppensetting impliziert, dass Kinder und Jugendliche die Möglichkeit haben, soziale Fertigkeiten interaktiv im direkten Kontakt zu erleben und zu erproben. In der Gruppe werden zwischenmenschliche Fertigkeiten und Integration aktiv gelebt. Zudem wird so das Gefühl gefördert, nicht alleine mit den „Problemen “ und belastenden Erfahrungen und Traumata zu sein. Wichtig ist, dass die Kinder und Jugendlichen selbst und freiwillig über die Teilnahme am STARTProgramm entscheiden. Als hilfreich hat sich bewährt, den Jugendlichen bereits vor Beginn des Interventionsprogramms ausführliche Informationen über Ziel und Ablauf der Module auszuhändigen. Materialien für ein entsprechendes Handout mit Informationen und Übungsblättern sind in übersetzter Sprache auf der DVD zum Ausdrucken bereitgestellt.

Die therapeutische Grundhaltung ist validierend und ressourcenaktivierend. Commitmentstrategien wie Cheerleading und Freiwilligkeit der Teilnahme am START-Programm zielen zudem auf die Förderung des Gefühls der Eigenkontrolle und der Selbstwirksamkeit bei Kindern und Jugendlichen, was insbesondere bei den erlebten Ohnmachts- und Hilflosigkeitserfahrungen traumatisierter Jugendlicher als bedeutungsvoll erscheint. START ist modular aufgebaut, die ersten vier Module sind auf die Vermittlung von Achtsamkeits- und Selbstberuhigungstechniken und Fertig- 
keiten zur Arousal-Modulation und Stressreduktion konzentriert.

Die ersten 4 Module beinhalten Übungen zur Stress- und Emotionsregulation, zur Selbstberuhigung und Selbstfürsorge. Achtsamkeitsübungen sind in allen Modulen berücksichtigt. Die vier Module können gut ohne Beteiligung eines Dolmetschers in der Gruppe umgesetzt werden, da auf visuelle, auditive und mehrsprachig übersetzte Materialien zurückgegriffen werden kann.

\section{Kurze Beschreibung der thematischen Schwerpunkte der einzelnen Module}

Modul 1 Einführung Achtsamkeit, Stressregulation und Entspannung: Konkrete Skills: Übungen und zu schnell wirksamen Hochstressskills wie zum Beispiel Kältereize (Coolpacks, Eiswürfel, kaltes Wasser), Psychoedukation und exemplarische Übung zu Bewegung und Sport und die Einführung zu selbstberuhigenden Skills „PMR“ in Kurzform

Modul 2 Übungen zu „mit den Sinnen beruhigen“ (Sehen, Riechen, Fühlen, Schmecken, Hören) als mögliche Regulationsfertigkeiten zum (Wieder-)
Erlangen von emotionaler und Verhaltenskontrolle im Hochstressbereich

Beispiele für Skills: Chilischoten oder Wasabipaste, Zitrone, Ammoniakampullen, Bilder, Musik, Bewegungübungen, antidissoziative Skills

Einführung der „Skillsbox“ (als Aufbewahrung von persönlichen Skills)

Modul 3 Vorstellen der bereits vorhandenen Skillsboxen

Zentrale Themen: „Achtsam mit sich selbst umgehen“; Anfertigen der „Persönlichen Skillsliste“ („Spannungsbogen“ exemplarische Skillsliste, Skillskette; „Spannungsbogen“)

Modul 4 Psychoedukation zum Thema Krise; interaktive Bearbeitung des Themas und Übungen mit TN zum Thema Krisen überwinden; Umgang mit Intrusionen und Flashbacks; neue Skillsübungen zu den Themen; Abschlussübung „Sicherer Wohlfühlort“

Modul 5 Dieses Modul ist fakultativ einsetzbar und enthält entgegen den Modulen 1 bis 4 expositionsbasierte bzw. narrative Aspekte. Es ist wichtig, dass bevor Modul 5 durchgeführt wird, bereits Stabilisierungstechniken und Skills etabliert sind. Außerdem
Abb. 4 Die wichtigsten Aspekte von START im Überblick

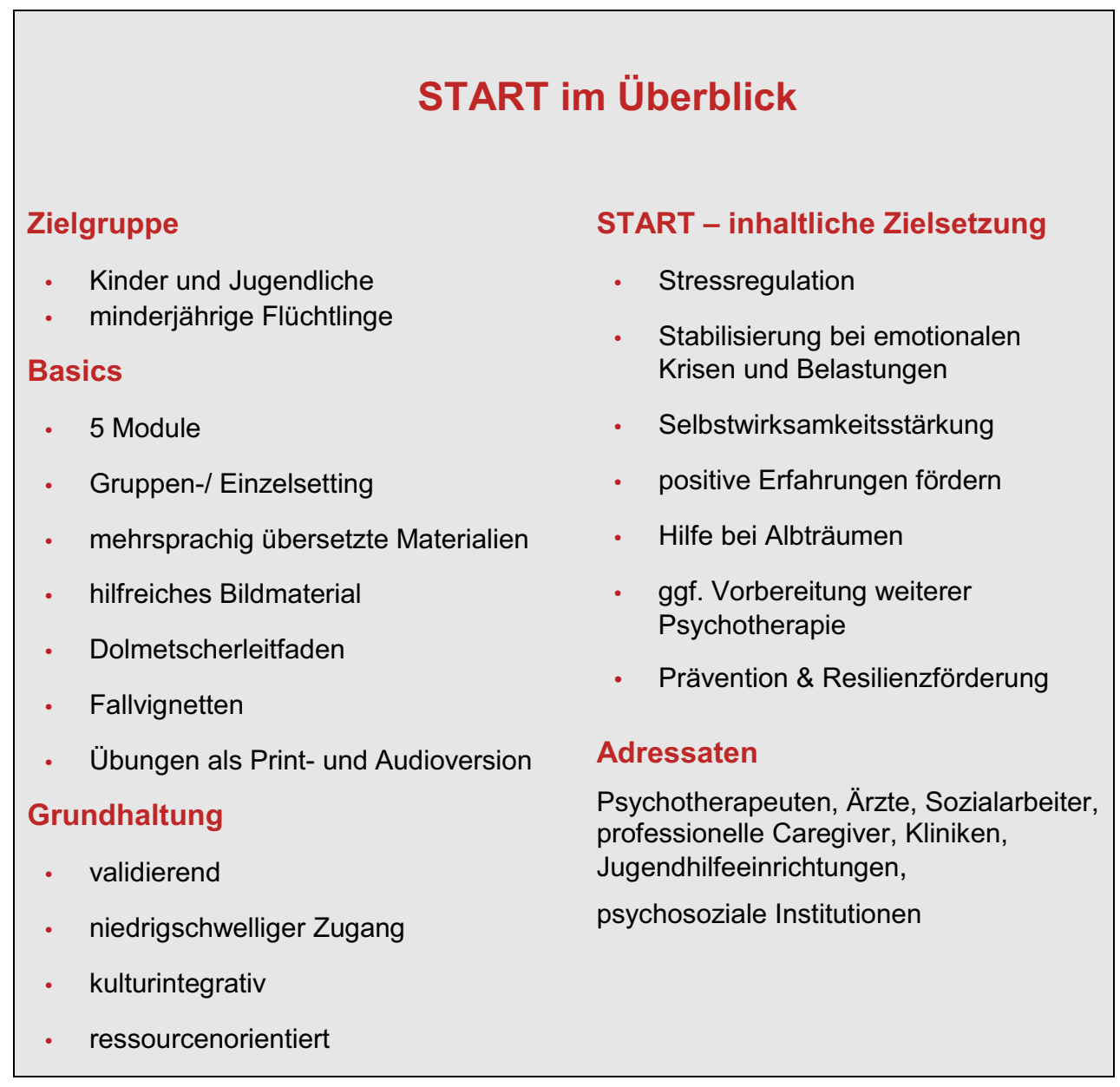


ist darauf zu achten, dass je nach Sprachkenntnissen des Jugendlichen der Einsatz eines Dolmetschers erforderlich sein kann. Zudem sollten Basics in Traumatherapie oder -pädagogik bei den durchführenden Therapeuten vorhanden sein.

Eine kurze Ablaufbeschreibung des Moduls:

1. Psychoedukation Alpträume;

2. Vorbereitung: an Skillsliste, „Persönlicher sicherer Wohlfühlort“ erinnern und geankerte „Geste“ zeigen lassen oder Schlüsselwort benennen lassen, antidissoziative Skills, Info - jederzeit STOP möglich; Einführung von Distanzierungstechniken

3. Übung: Alptraum erzählen lassen mir detaillierten Wahrnehmungen

4. Während der Übung (genauern von Kognitionen, Emotionen, Wahrnehmung)

5. schriftliche oder auditive Aufzeichnung des Alptraums

6. Modifikation des Alptraums mit Auseinandersetzung eines anderen, positiven Traumverlaufs

7. Fortführung in weiteren Therapiesitzungen und/ oder in Terminen mit geschulten pädagogischen Betreuern

Zusammenfassend sollen im fünften Modul Hilfen bei quälenden und wiederkehrenden Alpträumen erarbeitet werden. Kinder und Jugendliche mit Traumaerlebnissen berichten häufig von Schlafstörungen und Alpträumen und haben dabei enormen Leidensdruck, häufig gekoppelt mit massiven Schlafstörungen. Neben der Psychoedukation zum Thema und der Verbindung mit bereits erlernten Skills und Entspannungsübungen, werden Techniken zur Alptraumbeschreibung angewendet und die Möglichkeit zur Modifikation von Alpträumen als Basis zur Veränderung erarbeitet.

\section{Diskussion}

Das Programm wurde sowohl in Einrichtungen der Jugendhilfe als auch im Kontext der Kinder und Jugendpsychiatrie während der Entwicklung erprobt und hatte sich als durchführbar erwiesen. Mittlerweile ist START ein fest etabliertes Gruppenangebot in unserer klinischen Arbeit und Jugendhilfesettings. Angesichts der hohen Prävalenz von PTSD bei UMA (Walg et al. 2016) und anderen stressbezogenen Störungen ist ein Einsatz vor Ort in der Jugendhilfe aus verschiedenen Gründen vorteilhaft: Die Stigmatisierung der Jugendlichen entfällt, ebenso die Belastung durch Arztkontakte. Besonders wichtig bleibt dabei die Betonung der Freiwilligkeit zur Teilnahme am STARTProgramm. Da der Gruppenkontext den Schwerpunkt auf die Vermittlung von Skills legt und keine tiefgehende Selbstexploration angestrebt wird, „muss“ sich der Jugendliche nicht selbst als leidend „outen“ - zudem die Erfahrung zeigt, dass nämlich für sehr viele, vor allem männliche UMAs, seelisch krank $\mathrm{zu}$ sein, ausgesprochen schambesetzt und tabuisiert ist.
Die Struktur und die validierende Grundhaltung im Kontext des Settings der START- Gruppe legitimieren bzw. enttabuisieren Wahrnehmung von Gefühlen und Anspannungszuständen. Zentral ist die validierende Grundhaltung der Therapeuten bzw. der professionellen Helfer, da dies bereits einen entlastenden Effekt im Erleben der Jugendlichen zu erzeugen scheint.

\section{Limitationen}

In der Pilotstudie zu START im Prä-Post-Design wird die Wirksamkeit im ambulanten und stationären Setting der SHG Kliniken für Kinder und Jugendpsychiatrie unter standardisierten Bedingungen derzeitig untersucht. Erste Ergebnisse $(n=12)$ wurden auf dem DGKJP-Kongress 2017 der Fachöffentlichkeit vorgestellt. Es zeigen sich grundlegend signifikante Ergebnisse in Bezug auf „Emotionskontrolle“ und „Rückzug" (Feel-KJ) sowie ein positiver Trend bezüglich der Steigerung bei den Skalen „sozialer Unterstützung“, und Verringerung auf den Skalen „Selbstabwertung“ und „Peserveration“. Zudem ergibt sich ein positiver Trend beim Anspannungsbarometer (RHS-15). Die Studie ist derzeitig fortlaufend.

Eine Evaluation in der Jugendhilfe wird zudem aktuell angestrebt. Die sofortige Evaluation mit Einführung des START-Programms wurde erschwert durch die Schwierigkeit, ein Einverständnis des Vormundes zu bekommen, da im Prozess des Vorclearings, der Vormund erst etabliert werden muss. Da im Herbst 2017 keine Quotenübererfüllung des Saarlandes mehr vorliegen wird, ist dann ein längerfristiges Arbeiten und evaluieren möglich, da die Jugendlichen nicht mehr verteilt werden.

Interessenkonflikt A. Dixius und E. Möhler geben an, dass kein Interessenkonflikt besteht.

Open Access Dieser Artikel wird unter der Creative Commons Namensnennung 4.0 International Lizenz (http:// creativecommons.org/licenses/by/4.0/deed.de) veröffentlicht, welche die Nutzung, Vervielfältigung, Bearbeitung, Verbreitung und Wiedergabe in jeglichem Medium und Format erlaubt, sofern Sie den/die ursprünglichen Autor(en) und die Quelle ordnungsgemäß nennen, einen Link zur Creative Commons Lizenz beifügen und angeben, ob Änderungen vorgenommen wurden.

\section{Literatur}

BAMF (2016). Bundesamt für Migration und Flüchtlinge. https://www.bamf.de/SharedDocs/Anlagen/DE/ Downloads/Infothek/Statistik/Asyl/aktuelle-zahlen-zuasyl-april-2016.pdf?_blob=publicationFile. Zugegriffen: 10. Juli2016.

BAMF (2017). Bundesamt für Migration und Flüchtlinge. http://www.bamf.de/DE/Fluechtlingsschutz/ AblaufAsylv/Erstverteilung/erstverteilung-node.html. Zugegriffen: 16. Apr. 2017.

Bohus, M., \& Wolf, M. (2011). Interaktives Skillstraining für Borderline-Patienten. Stuttgart New York: Schattauer. 
Bryant, R. A., Harvey, A., Dang, S. T., Sackville, T., \& Basten, C. (1998). Treatment of acute stress disorder: a comparison of cognitive-behavioral therapy and supportive counseling. Journal of consulting and clinical Psychology, 66(5), 862-866.

Cohen, J.A., Mannarino, A. P., \& Deblinger, E. (2009). Traumafokussierte kognitive Verhaltenstherapie bei Kindern und Jugendlichen. Heidelberg: Springer.

Dixius, A., \& Moehler, E. (2017). Komplexe Krisenintervention bei einem 16-jährigen schwangeren Mädchen nach unbegleiteter Flucht aus Eritrea. Z Kinder Jugendpsychiatr Psychother, 45(1),69-74. doi:10.1024/1422-4917/a000458.

Gilbert, P. (2013). Compassion focused therapy. Paderborn: Jungfermann.

Hodes, M., Jagdev, D., Chandra, N., \& Cunniff, A. (2008). Risk and resilience for psychological distress amongst unaccompanied asylum seeking adolescents. Child Psychol Psychiatry, 49(7), 723-732. doi:10.1111/j.1469-7610.2008. 01912.x.

Huemer, J., Karnick, N. S., Voelkl-Kernstock, S., Granditsch, E., Dervic, K., Friedrich, M.H., et al. (2009). Mental health issues in unccompainied refugee minors. Child Adolesc Psychiatry Ment Health, 3(1), 13. doi:10.1186/1753-20003-13.

Krüsmann, M. (2003). Prävention posttraumatischer Störungen im Einsatzwesen. In W. Butollo \& M. Hagl (Hrsg.), Trauma, Selbst und Therapie. Konzepte und Kontroversen in der Psychotraumatologie (S. 147-161). Bern: Hans Huber.

Krüsmann, M., \& Müller-Cyran, A. (2005). Trauma und frühe Interventionen. München:Pfeiffer.

Miller, G. E., Chen, E., \& Parker, K. J. (2011). Psychological stress in childhood and suscep-tibility to the chronic diseases of aging: moving toward a model of behavioral and biological mechanisms. Psychol Bull, 137(6), 959-997. doi:10.1037/ a0024768.
Moehler, E., Simons, M., Kölch, M., Herpertz-Dahlmann, B., Schulte-Markwort, M., \& Fegert, J. (2015). Diagnosen und Behandlung (unbegleiteter) minderjähriger Flüchtlinge. Zeitschrift für Kinder- und Jugendpsychiatrie und Psychotherapie, 43(6), 381-383.

Mollica, R.F., McInnes, K., Poole, C., Tor, S. (1998). Doseeffect relationships of trauma to symptoms of depression and post-traumatic stress disorder among Cambodian survivors of mass violence. BrJ Psychiatry, 173, 482.

Linehan, M.M. (1996b). Dialektisch-Behaviorale Therapie der Borderline-Persönlichkeitsstörung. Trainingsmanual.München: CIP-Medien.

Linehan, M. M. (2015). DBT skills training manual. Ney York: Guilford Press.

Rathus, J.H., \& Miller, A. (2015). DBT skills manual for adolescents. New York: Guiford Press.

Rutter, M. (2000). Psychosocial influences: critiques, findings, and research needs. Dev Psychopathol, 12(3), 375-405.

Rutter, M., \&Sroufe, L. A. (2000). Developmental psychopathology: concepts and challenges. Dev Psychopathol, 12(3), 265-296.

Save The Children (2017). Unsichtbare Wunden. https:// assets.savethechildren.ch/downloads/invisible wounds_report_ch_low.pdf.Zugegriffen:10. März2017.

Thünker, J., \& Pietrowsky, R. (2011). Alpträume. Ein Therapiemanual. Göttingen:Hogrefe.

Walg, M., Fink, E., Großmeier, M., Temprano, M., \& Hapfelmeier, G. (2016). Häufigkeit psychischer Störungen bei unbegleiteten minderjährigen Flüchtlingen in Deutschland. Z Kinder Jugendpsychiatr Psychother, 45(1), 58-68. doi:10.1024/1422-4917/a000459.

Witt,A., Rassenhofer, M., Fegert, J., \&Plener, P. I. (2015).Hilfebedarf und Hilfeangebote in der Versorgung von unbegleiteten minderjährigen Flüchtlingen. Eine systematische Übersicht. Kindheit und Entwicklung, 24,209-234. 